\title{
Avaliação da capacidade de acolhimento da demanda espontânea nos serviços de atenção básica
}

\section{Assessment of the reception capacity of spontaneous demand in primary care services Evaluación de la capacidad de acogida de la demanda espontánea en los servicios de atención primaria}

Recebido: 09/11/2015 Aprovado: 29/02/2016 Publicado: 01/05/2016
Maria Cristina Traldi 1 Laís Rabesco ${ }^{2}$ Márcia Regina Campos Costa da Fonseca ${ }^{3}$

O objetivo do estudo foi avaliar o acolhimento dos serviços de atenção básica a partir das fichas classificadas como não urgência na escala de Manchester. Estudo exploratório com amostra aleatória constituída por 384 fichas azuis e verdes de crianças e adolescentes atendidos em serviço público de emergência. 0 endereço inscrito nas fichas possibilitou identificar a Unidade Básica da região de residência dos pacientes. 20,6\% tinham classificação verde e $79,4 \%$ azul. A mediana foi igual a oito atendimentos por Unidade de Atenção Básica. Vinte Unidades (55,5\%) obtiveram escore igual ou inferior à mediana, sendo considerados moderados na capacidade de acolhimento e $16(44,5 \%)$ ficaram acima da linha de corte e foram considerados como tendo baixa capacidade de acolhimento. 0 estudo concluiu que a capacidade de acolhimento de crianças e adolescentes foi moderada, com melhor desempenho das unidades que atuam na Estratégia Saúde da Família. Descritores: Atenção primária à saúde; Acesso aos serviços de saúde; Humanização da assistência.

This study's aim was to evaluate the receptivity of primary health care services, based on the records classified as non-urgent on the Manchester scale. Exploratory study with random sample consisted of 384 blue and green records of children and adolescents attended by public emergency services. The address entered on the records allowed the identification of the primary care service of the patients' region of residency. $20.6 \%$ were classified as blue and $79.4 \%$ as green. The median was equal to eight calls per Unit of Primary Care. Twenty units $(55.5 \%)$ had a score equal to or lower than the median, and were considered moderate in terms of receptivity and $16(44.5 \%)$ were above the cut line and were considered to have low receptivity. The study found that the receptivity of children and adolescents was considered mostly moderate, with better performance of the units working in the Family Health Strategy.

Descriptors: Primary health care; Health services accessibility; Humanization of assistance.

El objetivo de este estudio fue evaluar lo acogimiento en los servicios de atención primaria de salud promedio de las fichas clasificado como ninguna urgencia en acuerdo con la escala Manchester. Estudio exploratorio con muestra aleatoria que consistió de 384 registros de niños y adolescentes atendidos en servicio gubernamental de emergencia y con clasificación en colores azul y verde. La dirección introducida en las fichas se identificó la ubicación de la región de la unidad básica en la región de residencia de los pacientes. 20,6\% tenían clasificación azul y $79,4 \%$ verde. El promedio fue de ocho atendimientos por unidad de atención primaria. Veinte unidades de salud $(55,5 \%)$ tuvieron una puntuación igual o inferior a la mediana y fueron considerados como tiendo moderado capacidad de acogida y $16(44,5 \%)$ por encima de la línea de corte y se consideraron con baja capacidad de acogimiento. El estudio encontró que la mayoría de los servicios de salud tenían moderado capacidad de acogimiento, con un mejor rendimiento de las unidades de trabajo en la Estrategia Salud de la Familia.

Descriptores: Atención primaria de salud; Accesibilidad a los servicios de salud; Humanización de la atención.

\footnotetext{
${ }^{1}$ Enfermeira. Especialista em Medicina Social Preventiva. Mestre e Doutora em Educação. Professora Adjunta do Curso de Graduação em Enfermagem e do Programa de Mestrado Acadêmico em Ciências da Saúde da Faculdade de Medicina de Jundiaí (FMJ). mcristraldi@gmail.com. Brasil.

${ }^{2}$ Enfermeira graduada pela Faculdade de Medicina de Jundiaí. lais-rbesco@hotmail.com. Brasil.

3 Enfermeira. Mestre em Farmacologia. Doutora em Ciências Médicas. Professora Adjunta de graduação e do Programa de Mestrado Acadêmico em Ciências da Saúde da Faculdade de Medicina de Jundiaí (FMJ) e Professora da Faculdade São Leopoldo Mandic - Campinas. fonseca100@uol.com.br. Brasil.
} 


\section{INTRODUÇÃO}

O Sistema Único de Saúde (SUS) instituído em $1990 \mathrm{com}$ a finalidade de alterar a situação de desigualdade no acesso e na qualidade da assistência à saúde da população brasileira, ainda mantém, nos dias atuais, o desafio de efetivar políticas que garantam a equidade no acesso aos serviços e a universalidade da assistência integral à saúde ${ }^{1}$.

Um dos pontos centrais das proposições do SUS na primeira década de sua implementação era a necessidade de promover uma mudança radical no modelo assistencial vigente. Naquele contexto, a atenção básica foi acertadamente, considerada como caminho para a universalização da assistência. No entanto, o que prevaleceu foi a focalização das ações preventivas, centradas no controle das doenças de maior prevalência e na assistência de baixa complexidade dirigidas à mulher e à criança ${ }^{2,3}$.

Para enfrentar a focalização das ações, foi instituído, em 1994, o Programa Saúde da Família (PSF), que se apresentava como alternativa para promover a mudança que não havia sido efetivada. Na expectativa desse processo ocorrer em larga escala, o programa assumiu o caráter de estratégia. Devido ao potencial estruturante que exerce sobre os sistemas de saúde municipais, a Estratégia de Saúde da Família (ESF) tem provocado um importante movimento de reordenação no modelo de atenção no SUS, pois busca maior racionalidade na utilização dos demais níveis assistenciais e tem produzido resultados pontuais positivos na avaliação de usuários, gestores e profissionais de saúde, no que se refere à oferta de ações de saúde e ao acesso e uso de serviços ${ }^{1,4}$, inclusive reduzindo a mortalidade infantil 5 .

Contudo, ainda permanece o desafio da efetividade das ações acompanhar em a expansão da rede, reduzindo o distanciamento entre as práticas recomendadas e aquelas efetivamente implementadas, tanto no que se refere ao ritmo quanto em proporção nos diferentes cenários que se apresentam nas regiões do país 6 .
Nas políticas públicas a expressão Atenção Básica é utilizada como sinônimo de Atenção Primária de Saúde, sendo definida como um conjunto de ações destinadas a promover e proteger a saúde da população, prevenir, diagnosticar e tratar agravos, reabilitar e manter a saúde de indivíduos e coletivos populacionais em territórios delimitados, ou seja, ações dirigidas a uma população adscrita a um serviço de saúde que, em geral, é uma unidade básica de saúde (UBS) ou uma unidade de saúde da família (USF) ${ }^{7}$.

$\mathrm{Na}$ perspectiva estruturante dos sistemas locais e do cuidado à saúde, a atenção primária é responsável pelo cuidado integral dos usuários, incluindo entre outros aspectos, $\mathrm{o}$ primeiro atendimento às urgências $\mathrm{e}$ emergências, constituindo-se no elo mais forte da relação com o usuário que, em suas necessidades de saúde, buscam algum tipo de resposta para as condições de vida, para assim, qualificar e prolongar sua existência ${ }^{1,2,4-7}$.

Para ser resolutiva e permitir acesso aos usuários, os serviços de atenção primária devem demonstrar capacidade de escutar e oferecer soluções para lidar com a complexa demanda de problemas e necessidades de saúde das populações, ou seja, demonstrar habilidade para realizar o acolhimento e ser capaz de dar respostas positivas aos usuários, seja inserindo-o em uma rede de atenção, solicitando apoio matricial de profissionais que possam subsidiar a equipe local. Nesse modelo de atenção, espera-se da atenção primária a resolução da maioria das necessidades de saúde dos indivíduos e coletivos que residam no território das unidades ${ }^{3,8}$.

A garantia do acesso universal àqueles que realmente demandam esse tipo de atendimento ainda não está consolidada no SUS, e as justificativas para as dificuldades na sua implementação são distintas passando, necessariamente, pela capacidade de absorção da demanda local dos serviços. Esta é influenciada pelo nível de tecnologia disponível, nível de informação dos usuários, influências que o complexo médico-industrial exerce sobre a sociedade e, sobretudo, pela 
oferta e capacidade de acolhimento e resolutividade dos serviços de atenção primária ${ }^{1}$.

Independente da justificativa, a consequência imediata à restrição no acesso às demandas não programadas na atenção primária é o aumento na procura por serviços de pronto atendimento, sobrecarregando um nível de assistência planejado para receber, fundamentalmente, casos de maior complexidade na condição de demanda referida, ordenada pela política de construção das redes de atenção e não por demanda espontânea ${ }^{8,9}$.

A sobrecarga nos serviços de pronto atendimento hospitalar desorganiza o sistema, sobrecarrega os serviços com demandas não urgentes e contribui com o aumento do tempo de espera do usuário por atendimento. Expõe, por outro lado, as falhas de implementação da política nacional de saúde que tem a atenção primária como elemento estruturante da rede de atenção à saúde e a abordagem da família como principal estratégia de ação do modelo de atenção integral à saúde ${ }^{6}$.

No intuito de reduzir o tempo de espera e equacionar o atendimento, os serviços públicos passaram a adotar medidas de acolhimento normatizadas em um protocolo para classificação de risco nos serviços de pronto atendimento. Este fornece parâmetros para identificar prioridades baseadas nas queixas referidas pelo usuário e nos sinais e sintomas apresentados no momento da avaliação. 0 protocolo estratifica em quatro níveis de gravidade, utilizando cores para identificar e priorizar o atendimento. Assim, aos casos considerados emergência, atribui-se tarja vermelha à ficha; às urgências o amarelo; aos casos considerados não urgência, o verde; e às necessidades de consultas de baixa complexidade, a ficha recebe a cor azul ${ }^{10}$.

Serviços de pronto socorro com grande demanda por atendimento classificado como não emergência, caracterizados pelas cores verde e azul, constituem indicador importante de baixa capacidade de acolhimento e de resolutividade da atenção primária ${ }^{11,12}$.
A procura espontânea por serviço de urgência e emergência com queixa não caracterizada como tal pode ser, dentre outros, um indicador de que o acolhimento e a resolutividade dos serviços de atenção primária estão em dissonância com as proposições do modelo assistencial, que preconizam o vínculo com responsabilização pela saúde no território de ação, a integralidade da atenção e a centralidade na coordenação da atenção à saúde no âmbito do SUS10,11.

A questão que norteou a realização desta pesquisa foi a de analisar a capacidade de acolhimento das demandas provenientes da população infantil e adolescente, por parte dos serviços de atenção primária. Com este foco, o estudo objetivou avaliar o acolhimento dos serviços de atenção básica a partir das fichas de atendimento de crianças e adolescentes classificadas como não urgência na escala de Manchester (verde e azul), em serviço de emergência de um hospital do SUS, num município do interior paulista.

\section{MÉTODO}

Trata-se de um estudo transversal e retrospectivo, realizado no pronto socorro de um hospital localizado em um município de médio porte, no interior do Estado de São Paulo, com aproximadamente 400 mil habitantes. O hospital é referência para os usuários do Sistema Único de Saúde nas especialidades materna e infantil e atende à demanda espontânea em sistema "porta aberta", ou seja, sem exigência de referenciamento.

A amostra foi calculada com base no número atendimento de crianças e adolescentes de 0 a 14 anos, moradores do município, atendidos no referido serviço de pronto socorro, no ano de 2013, em dias úteis e em horário comercial. 0 critério de horário e dias visou garantir que os atendimentos ocorreram em horário de funcionamento das unidades de saúde de atenção primária.

0 município em que ocorreu o estudo localiza-se na região Sudeste do Estado de São 
Paulo e dista, aproximadamente, 60 quilômetros da capital paulista. Tem população estimada para 2015 de 400 mil habitantes (IBGE Cidades, 2010: www.cidades.ibge.gov.br/xtras/perfil.php?lan g=\&codmun=352590), dos quais cerca de 4.500 são crianças com menos de um ano de idade, 18.000 na faixa de um a quatro anos e 59.000 entre cinco a 14 anos de idade, totalizando população infantil de 81.500 habitantes. 0 Índice de desenvolvimento Humano do município IDHM é de 0,822 e integra a faixa de mais alto nível do país.

A amostra foi calculada, assumindo a maior variabilidade possível, baseada na prevalência $\mathrm{p}=50 \%$, um nível de significância de $5 \%$ e um erro amostral de 5\%, considerando 5500 atendimento/mês, gerando $\mathrm{n}=384$.

Para a estratificação da amostra, foi aplicado o percentual de $0,6 \%$ em cada mês do ano para obter o número de fichas a serem eleitas.

A aleatorização foi garantida com a escolha do terceiro de cada três dias $(3 ; 6 ; 9$; $12 ; . .$.$) , excluindo-se os sábados, domingos e$ feriados. A eleição das fichas seguiu a mesma sistemática da seleção dos dias, adotando-se a terceira ficha de cada três, seguindo a ordem crescente de numeração das mesmas até a obtenção do número necessário para completar o percentual proporcional mensal da amostra.

As variáveis sociodemográfica de interesse foram: endereço residencial; idade e sexo; as variáveis para a caracterização do acolhimento foram: a queixa principal, a classificação de risco e desfecho; as variáveis de caracterização do serviço de atenção primária: tipo de Unidade (UBS/USF) e o horário do atendimento.

Em decorrência da diversidade de queixas adotou-se uma categorização para agrupá-las de acordo com grupo de sintomas e/ou a proximidade aos principais sistemas do corpo humano: sistema respiratório, digestório, geniturinário, tegumentar. Devido à alta frequência entre os motivos de procura pelo serviço de pronto socorro, a febre inespecífica, isolada ou associada a outros sintomas, constituiu um grupo de queixa.

A identificação do endereço de residência da criança ou adolescente com a área de abrangência das UBS \USF foi feita com base na lista de ruas e bairros disponibilizada pela Secretaria Municipal de Saúde local.

Para efeito deste estudo, considerou-se como unidades que adotam a estratégia de saúde da família, as USF e as UBS que contam com Programa de Agentes Comunitário de Saúde (PACS). No período do estudo os serviços de atenção primária somavam 36 unidades, sendo 18 USFs e 18 UBSs, localizados prioritariamente na periferia da cidade.

Os dados, coletados em 2014, foram digitados em planilha Excel e passaram por análise descritiva para cálculo das medidas de tendência central e de dispersão. Para avaliar a capacidade de acolhimento das unidades foi construído um escore definido a partir da mediana de frequência de atendimentos da amostra. Unidades com escore de fichas atendidas abaixo da média foram incluídas na categoria moderada capacidade de acolhimento; as que se posicionaram acima da média, com baixa capacidade de acolhimento.

A opção pela classificação da capacidade de acolhimento em moderada ou baixa se justificou na elevada demanda por atendimento de eventos sem características de urgência ou emergência no serviço estudado. De acordo com os relatórios do Pronto Socorro Infantil (PSI), no ano de 2013 foram realizados 63.569 consultas médicas e adolescentes, destes, $1.190 \quad(1,87 \%)$ permaneceram internados. Percentual muito inferior aos $6 \%$ a 7\% registrados no Pronto Socorro de PelotasRS, produzido pelo Conselho Municipal de Saúde do Município de Pelotas em 2007 com dados de 2006 (www.pelotas.rs.gov.br/cmspel/relatorio_psp. $p d f)$.

O estudo foi submetido e aprovado no Comitê de Ética da Faculdade de Medicina de Jundiaí sob o número 045535/2014. Por se tratar de estudo retrospectivo, com base em 
dados secundários, foi dispensado o Termo de Consentimento Livre e Esclarecido.

\section{RESULTADOS}

A amostra deste estudo $(n=384)$ foi obtida das fichas de atendimento (FA) dos pacientes que passaram efetivamente por atendimento, pois alguns usuários que chegam ao serviço de pronto atendimento preenchem a ficha e não aguardam por atendimento médico. No ano de 2013, o serviço estudado teve percentual de abandono "não aguardou atendimento" de $1,11 \%(n=715)$.

A amostra foi composta por fichas de atendimento de 384 crianças e adolescentes $(0$ a 14 anos de idade). 0 perfil sociodemográfico indica que a média de idade dos amostrados foi de 4,47 \pm 4,13 anos, com variação de 12 dias a 14 anos de idade, sendo mais frequente $(45,8 \%)$ o atendimento de crianças na faixa etária de 01 a 04 anos.

As fichas amostradas resultaram nos seguintes percentuais por classificação de risco: verde $20,57 \%$ (79/384), azul $73,95 \%$ (284/384), amarelo 5,2\% (20/384) e vermelho $0,26 \%$ (1/384), com $94,52 \%$ de atendimentos classificados na categoria de não urgência.

Quanto ao desfecho, 7,29\% (28/384) permaneceram em observação do pronto socorro, sem que destes resultasse alguma internação; os demais (356/384) foram dispensados após consulta médica.

Os meses que apresentaram maior demanda por atendimento no pronto socorro infantil foram maio $(11,7 \%)$ e abril $(10,7 \%)$. Credita-se a maior frequência à influência da sazonalidade da demanda associada à mudança no clima, característico da estação do outono na qual as temperaturas tendem a cair e os ambientes permanecem com pouca ventilação, propiciando a transmissão de vírus por aerossóis e o consequente aumento das queixas respiratórias.

0 intervalo de horas que concentrou maior número de acolhimento foi o de início da tarde, entre $14 \mathrm{~h} 00$ e $15 \mathrm{~h} 00$, sendo o período vespertino de maior frequência $(60,2 \%)$ de atendimentos (Gráfico 1). Vale destacar que o horário de funcionamento das UBS/USF para consultas no município é das $08 \mathrm{~h} 00$ às $17 \mathrm{~h} 00$, salvo aquelas que funcionam em horário estendido ( $3^{\circ}$. Turno).

A média mensal de fichas de atendimentos realizados no PSI por unidade de atenção primária de origem foi 10,97 \pm 9,87 fichas por serviço (2-53); uma unidade não registrou atendimento no PSI-HU.

Gráfico 1. Distribuição da amostra por horário do atendimento, São Paulo, 2013.

\section{$n=384$ Atendimentos}

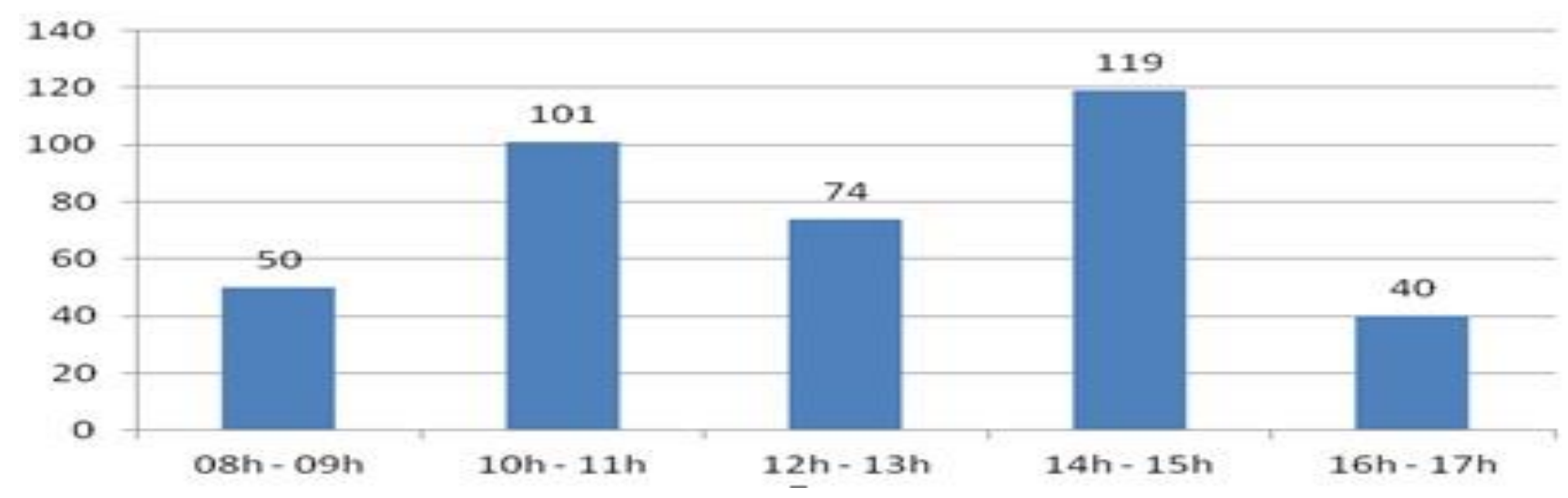

As queixas mais frequentemente registradas nas fichas de atendimento foram as dos grupos G1 e G2, que incluem febre isolada

ou associada e sintomas característicos de doenças do sistema respiratório como tosse e coriza. Juntas, as queixas desses dois grupos 
concentraram $\quad 47,14 \%$ dos atendimentos as faixas etárias (Tabelas 1 e 2). realizados no serviço de emergência, em todas

Tabela 1. Frequência absoluta e relativa das queixas registradas nas fichas de atendimento, por grupo, São Paulo, 2013.

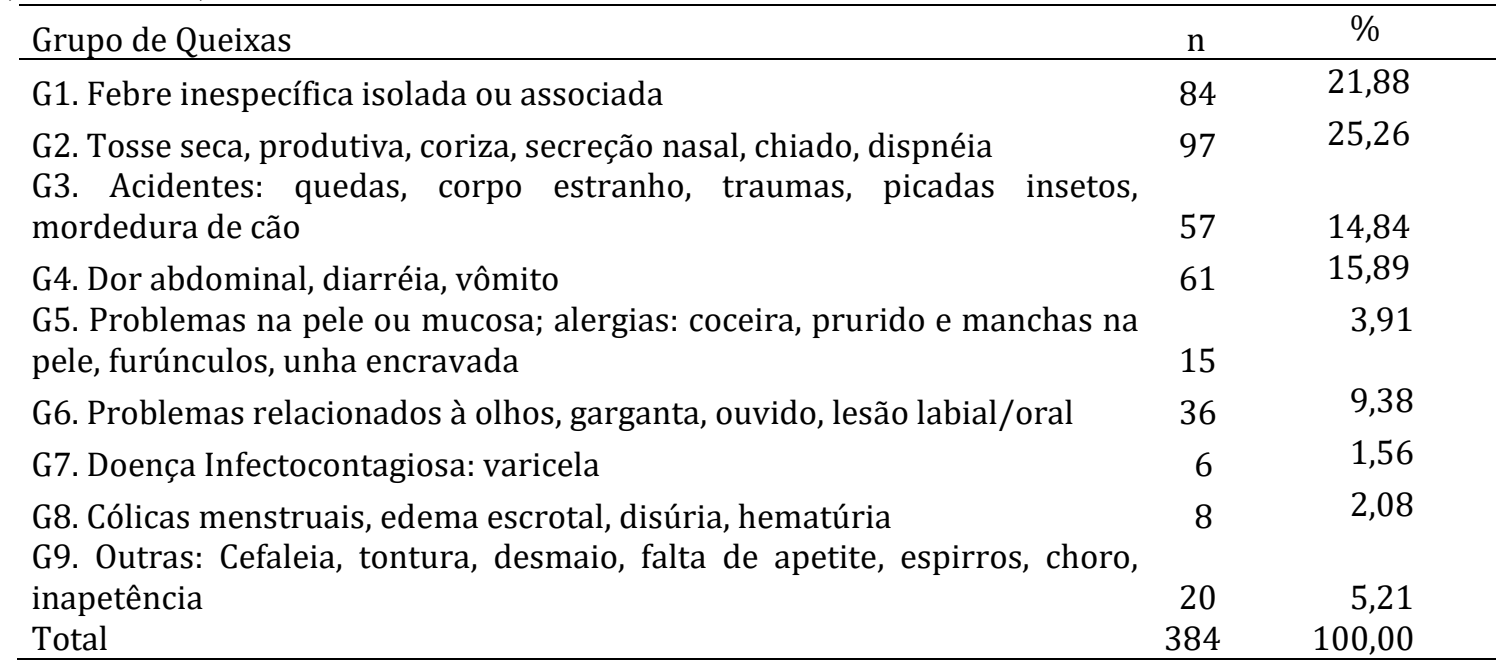

Tabela 2. Frequência absoluta e relativa das queixas agrupadas e faixa etária, São Paulo, 2013.

\begin{tabular}{lrrrrrrrr}
\hline Queixas & \multicolumn{2}{c}{$<$ ano } & \multicolumn{2}{c}{$1-4$ anos } & \multicolumn{2}{c}{$5-9$ anos } & \multicolumn{2}{c}{$10-14$ anos } \\
\hline & $\mathrm{n}$ & \multicolumn{1}{c}{$\%$} & \multicolumn{1}{c}{$\mathrm{n}$} & \multicolumn{1}{c}{$\%$} & \multicolumn{1}{c}{$\mathrm{n}$} & \multicolumn{1}{c}{$\%$} & $\mathrm{n}$ & \multicolumn{1}{c}{$\%$} \\
\hline G1 & 19 & 28,79 & 46 & 26,14 & 16 & 57,50 & 3 & 4,84 \\
G2 & 26 & 39,39 & 42 & 23,86 & 18 & 52,50 & 21 & 33,87 \\
G3 & 4 & 6,06 & 30 & 17,05 & 11 & 37,50 & 2 & 3,23 \\
G4 & 4 & 6,06 & 24 & 13,64 & 20 & 30,00 & 13 & 20,97 \\
G5 & 4 & 6,06 & 6 & 3,41 & 2 & 7,50 & 3 & 4,84 \\
G6 & 5 & 7,58 & 20 & 11,36 & 6 & 25,00 & 5 & 8,06 \\
G7 & & 0,00 & 4 & 2,27 & 2 & 5,00 & & 0,00 \\
G8 & 1 & 1,52 & & 0,00 & 1 & 0,00 & 6 & 9,68 \\
G9 & 3 & 4,55 & 4 & 2,27 & 4 & 5,00 & 9 & 14,51 \\
Total & 66 & 100,00 & 176 & 100,00 & 80 & 100,00 & 62 & 100,00 \\
\hline
\end{tabular}

A mediana de atendimentos classificados na escala de Manchester nas cores verde e azul foi oito. $\mathrm{Na}$ avaliação da capacidade de acolhimento, 20 (55,5\%) Unidades de Saúde obtiveram escore igual ou inferior à mediana, sendo considerados moderados na capacidade de acolhimento e 16 $(44,5 \%)$ ficaram posicionados acima da linha de corte e foram considerados neste estudo com baixa capacidade de acolhimento.

Juntas, as 14 Unidades de Saúde com baixa capacidade de acolhimento responderam por $70,6 \%$ (271/384) dos atendimentos com classificação Manchester azul e verde no PSI; seis são UBS, oito são UBS que contam com apoio do programa de agentes comunitários de saúde (PACS), além de uma unidade identificada como UBS/ESF, cujos bairros que compõem a área de abrangência são parte de uma mesma região com uma única denominação e não puderam ser identificados nas fichas de atendimento (Tabela 3).

Destaque para o fato de que as cinco unidades que atuam na ESF estão incluídas entre aquelas que possuem moderada capacidade de acolhimento e todas com escore abaixo da mediana. 
Tabela 3. Amostra por faixa etária e tipo de Unidade de atenção primária, São Paulo, 2013.

\begin{tabular}{|c|c|c|c|c|c|c|c|c|c|c|}
\hline \multirow[t]{2}{*}{ Tipo Unidade } & \multicolumn{2}{|c|}{ Atendimento } & \multicolumn{8}{|c|}{ Faixa Etária } \\
\hline & $\mathrm{n}$ & $\%$ & $<1$ a. & $\%$ & $1-4$ & $\%$ & $5-9$ & $\%$ & $10-14$ & $\%$ \\
\hline Total & 384 & 100 & 66 & 17,2 & 176 & 45,8 & 80 & 20,8 & 62 & 16,1 \\
\hline UBS & 4 & 2,7 & 2 & 3,0 & 1 & 0,6 & & & 1 & 1,6 \\
\hline UBS & 6 & 4 & & 0,0 & 2 & 1,1 & 2 & 2,5 & 2 & 3,2 \\
\hline UBS & 4 & 2,7 & 1 & 1,5 & 3 & 1,7 & & 0 & & 0,0 \\
\hline UBS & 4 & 2,7 & & 0,0 & 1 & 0,6 & 1 & 1,25 & 2 & 3,2 \\
\hline UBS & 9 & 6 & 1 & 1,5 & 4 & 2,3 & 2 & 2,5 & 2 & 3,2 \\
\hline UBS & 3 & 2 & & 0,0 & 2 & 1,1 & 1 & 1,25 & & 0,0 \\
\hline UBS & 7 & 4,7 & 2 & 3,0 & 3 & 1,7 & 2 & 2,5 & & 0,0 \\
\hline UBS & 4 & 2,7 & & 0,0 & 3 & 1,7 & 1 & 1,25 & & 0,0 \\
\hline UBS & 5 & 3,4 & 2 & 3,0 & 1 & 0,6 & 1 & 1,25 & 1 & 1,6 \\
\hline UBS & 20 & 13,4 & 4 & 6,1 & 9 & 5,1 & 5 & 6,25 & 2 & 3,2 \\
\hline UBS & 13 & 8,7 & 1 & 1,5 & 7 & 4,0 & 3 & 3,75 & 2 & 3,2 \\
\hline UBS & 12 & 8,1 & & 0,0 & 8 & 4,5 & 3 & 3,75 & 1 & 1,6 \\
\hline UBS & 13 & 8,7 & 3 & 4,5 & 5 & 2,8 & 3 & 3,75 & 2 & 3,2 \\
\hline UBS & 8 & 5,4 & 1 & 1,5 & 3 & 1,7 & 3 & 3,75 & 1 & 1,6 \\
\hline UBS & 13 & 8,7 & 4 & 6,1 & 4 & 2,3 & 3 & 3,75 & 2 & 3,2 \\
\hline UBS & 8 & 5,4 & 1 & 1,5 & 4 & 2,3 & 3 & 3,75 & & 0,0 \\
\hline UBS & 16 & 10,7 & 3 & 4,5 & 6 & 3,4 & 4 & 5 & 3 & 4,8 \\
\hline UBS+PACS & 13 & 7,3 & & 0,0 & 7 & 4,0 & 5 & 6,25 & 1 & 1,6 \\
\hline UBS+PACS & 2 & 1,12 & & 0,0 & 1 & 0,6 & 1 & 1,25 & & 0,0 \\
\hline UBS+PACS & 3 & 1,69 & & 0,0 & 2 & 1,1 & & 0 & 1 & 1,6 \\
\hline UBS+PACS & 8 & 4,49 & 2 & 3,0 & 5 & 2,8 & & 0 & 1 & 1,6 \\
\hline UBS+PACS & 3 & 1,69 & & 0,0 & 2 & 1,1 & & 0 & 1 & 1,6 \\
\hline UBS+PACS & 15 & 8,43 & 2 & 3,0 & 8 & 4,5 & 2 & 2,5 & 3 & 4,8 \\
\hline UBS+PACS & 21 & 11,8 & 1 & 1,5 & 12 & 6,8 & 5 & 6,25 & 3 & 4,8 \\
\hline UBS+PACS & 53 & 29,78 & 11 & 16,7 & 22 & 12,5 & 9 & 11,25 & 11 & 17,7 \\
\hline UBS+PACS & 11 & 6,18 & 2 & 3,0 & 6 & 3,4 & 2 & 2,5 & 1 & 1,6 \\
\hline UBS+PACS & & 0 & & 0,0 & & 0,0 & & 0 & & 0,0 \\
\hline UBS+PACS & 23 & 12,92 & 5 & 7,6 & 7 & 4,0 & 7 & 8,75 & 4 & 6,5 \\
\hline UBS+PACS & 8 & 4,49 & 1 & 1,5 & 6 & 3,4 & & 0 & 1 & 1,6 \\
\hline UBS+PACS & 18 & 10,11 & 4 & 6,1 & 9 & 5,1 & 2 & 2,5 & 3 & 4,8 \\
\hline UBS/PSF & 30 & 52,63 & 5 & 7,6 & 13 & 7,4 & 6 & 7,5 & 6 & 9,7 \\
\hline USF & 7 & 12,28 & 3 & 4,5 & 4 & 2,3 & & 0 & & 0,0 \\
\hline USF & 7 & 12,28 & 3 & 4,5 & 2 & 1,1 & & 0 & 2 & 3,2 \\
\hline USF & 6 & 10,53 & 1 & 1,5 & 1 & 0,6 & 3 & 3,75 & 1 & 1,6 \\
\hline USF & 2 & 3,51 & & 0,0 & & 0,0 & 1 & 1,25 & 1 & 1,6 \\
\hline USF & 5 & 8,77 & 1 & 1,5 & 3 & 1,7 & & 0 & 1 & 1,6 \\
\hline
\end{tabular}

DISCUSSÃO

Os resultados deste estudo remetem à reflexão sobre o impacto da assistência básica e em especial da estratégia saúde da família, em promover o reordenamento do modelo assistencial a partir do acolhimento dos usuários. 0 pressuposto é o de que o vínculo estabelecido entre profissionais da equipe de 
saúde local e a população efetiva a atenção primária como porta de entrada para o sistema, passando a ser a principal referência no atendimento das necessidades de saúde de baixa complexidade, dos usuários pertencentes a um determinado território.

Desde as primeiras tentativas de implantar um modelo de assistência que pudesse provocar a alteração na maneira de organizar o sistema de saúde a partir da hierarquização da atenção integral, o atendimento das demandas programadas tem sido a marca da produção em saúde na atenção primária, privilegiando a assistência pré-natal, a puericultura, a imunização, o controle da tuberculose, da hanseníase e de outras doenças transmissíveis endêmicas ou epidêmicas ${ }^{15}$.

Portadores de doenças crônicas como hipertensão arterial, diabetes mellitus, transtornos psiquiátricos, assim como o atendimento odontológico, foram progressivamente integrados ao cotidiano da assistência. Contudo, a ampliação do espectro assistencial continua priorizando os atendimentos programados e atendendo a um modelo de produção de ações que se alinha mais com um tipo de gestão baseada no controle dos processos em detrimento do resultado, contrariando as novas políticas de gestão pública ${ }^{15}$.

0 acolhimento dos usuários em suas necessidades de saúde não programadas passa pelo estabelecimento de um fluxo de atendimento iniciado pela escuta daqueles que chegam ao serviço buscando uma solução para o problema de saúde ${ }^{16,17}$. Essa atitude da equipe favorece a gestão por resultados na medida em que busca atender o usuário em sua integralidade, e assim, converge em direção à proposta de humanização e contribui para efetivar o acesso ao sistema pela porta de entrada da atenção primária.

No município estudado, 47,2\% (17/36) das unidades de saúde de atenção primária atuam na perspectiva da ESF, seja como equipes de saúde da família ou com PACS. As USF se posicionaram entre as unidades com moderada capacidade de acolhimento e uma delas não teve ficha de atendimento amostrada neste estudo.

Os resultados sugerem melhor capacidade de acolhimento das equipes que atuam na ESF, comparada ao das UBS e reafirma a política pública da ESF adotada em âmbito nacional, como reorientadora do modelo de assistência para melhorar o acesso da população à atenção primária ${ }^{13}$. Corrobora outros estudos que apontam serem o acolhimento e o vínculo as principais mudanças verificadas nas unidades de saúde que atuam na estratégia da saúde da família, quando o foco da análise é a demanda,17-19.

As queixas registradas nas fichas de atendimento que levaram pais e/ou responsáveis por crianças e adolescentes ao serviço de PSI sugerem que o acolhimento da demanda espontânea nos serviços de atenção primária não está sendo efetivo em sua totalidade, tal como se verifica em outras localidades ${ }^{20}$.

0 acompanhamento programático de crianças com menos de um ano de idade nas unidades de saúde, com agendamento de retornos periódicos são oportunidades para o atendimento das demandas espontâneas para este grupo populacional e pode explicar a menor frequência de procura no pronto socorro por esse grupo populacional, que registrou um dos menores percentuais de atendimentos realizados por queixas caracterizadas como não urgência. Crianças pequenas na faixa etária entre 1 a 4 anos, ao contrário, foram as que mais buscaram os serviço de emergência e são justamente as que não mais desfrutam da agenda de retornos programados na frequência dos lactentes.

Assumir efetivamente o acolhimento como diretriz é um processo que demanda transformações intensas na maneira de pensar e atuar na atenção primária. Requer um conjunto de ações articuladas, envolvendo usuários, trabalhadores e gestores, pois a implantação do acolhimento dificilmente se dá apenas a partir da vontade de um ator isolado. Com efeito, múltiplos aspectos técnicos e político-institucionais precisam ser 
mobilizados para o êxito de sua implementação ${ }^{18}$.

As políticas públicas de saúde e de humanização referem que acolher na atenção primária é uma maneira objetiva de identificar, reconhecer e intervir sobre as demandas que chegam do usuário, aceitando o fato de que este também define necessidades de saúde em formas e graus variados.

Nessa concepção da humanização da assistência, o acolhimento deve ser compreendido como um dispositivo potente para a mudança no processo de trabalho que propicie o vínculo entre a equipe de saúde e a população e contribua para aumentar as atitudes fundamentadas na ética da responsabilidade institucional e dos trabalhadores pelo cuidado integral dos usuários no território de atuação.

0 acolhimento, na prática, se implementa a partir da recepção do usuário por meio da escuta qualificada da queixa e segue com a garantia de atenção resolutiva da necessidade de saúde, desencadeando uma ação local imediata ou agendada na própria unidade, dependendo do caso, ou em articulação com outros serviços de saúde, para a continuidade da assistência ${ }^{10}$.

Algumas queixas que levaram pais/responsáveis a buscar atendimento em serviço de emergência podem refletir desconhecimento a respeito da finalidade de um serviço de pronto socorro, dos princípios fundamentais do SUS e da resolutividade esperada para os serviços de atenção primária. Esta afirmação pode ser válida para todas as queixas classificadas nas cores verde e azul, mas em especial para as incluídas no grupo "outras" no qual se identificou a falta de apetite como motivo de busca por atendimento no PSI. Por outro lado, pode refletir o descrédito na possibilidade de conseguir atendimento para uma necessidade de saúde identificada pelo usuário.

Nesse aspecto é importante destacar que a escuta acurada e o efetivo acolhimento das demandas espontâneas, legitima e reconhece o problema que motivou a procura do serviço, independente de haver convergência ou não entre a percepção de necessidade do usuário e da equipe de saúde.

Há que se estabelecer um esforço de diálogo e compreensão, sem o qual são produzidos ruídos que se materializam por vezes em retornos repetidos ou busca por outros serviços, como os de emergência, na tentativa de conseguir um atendimento, na medida em que o sistema de saúde não se organiza para isso ${ }^{12}$. Deixar de acolher pela ausência de recepção e escuta do usuário não só empobrece o processo assistencial suprimindo deste o componente da longitudinalidade como também desrespeita as necessidades individuais do usuário ${ }^{21}$.

Cabe destacar que o atendimento à demanda espontânea é, também, uma oportunidade para as equipes avaliarem a efetividade de algumas condutas e projetos terapêuticos prévios, ou se deparar com situações que requerem invenção de novas estratégias de cuidado e de reorganização do processo de trabalho, constituindo-se em um dispositivo importante de reorientação do modelo de atenção, pois a rede básica de serviços de saúde não tem conseguido se tornar a principal porta de entrada para o sistema de saúde ${ }^{12}$.

A avaliação de indicadores de resultado oportuniza as equipes locais, elaborarem o diagnóstico situacional e analisar criticamente seus resultados, avaliando as metas estabelecidas e comparando os resultados com outras unidades congêneres, reorientando as ações com base na proposta de modelo assistencial, caso seja necessário.

$\mathrm{Na}$ avaliação para o planejamento do trabalho em saúde, além de preparo dos recursos humanos para atender na perspectiva da integralidade 22 , outros aspectos da organização do sistema são apontados como fragilidades que podem comprometer o acesso dos usuários como a falta de articulação com os outros níveis de atenção à saúde; a capacidade instalada da unidade no que se refere à composição da equipe multidisciplinar, assim como a falta de políticas 
intersetoriais no âmbito do município que viabilizam a concretização das ações de promoção da saúde da população local, pois reduzem as tentativas de tomadas de atitude pontuais e voluntaristas da equipe de saúde local que, muitas vezes, age em face da ausência de ação dos gestores municipais ${ }^{23-25}$.

Agir nestas frentes para alterar a perspectiva de assistência e reorientar o processo de trabalho das equipes para garantir o acesso e melhorar a resolutividade da atenção primária é ainda um desafio a ser enfrentado pelos gestores, profissionais de saúde e o conjunto da sociedade.

Estudos revelam que as mudanças no modelo de atenção no país ainda são incipientes e resultam de avanços pontuais, não podendo ser atribuídos à adoção da Saúde da Família como estratégia prioritária e central no nível local ${ }^{13,26}$. Destacam que apesar das políticas que visam melhorar o acesso e o acolhimento, ainda permanece a dificuldade em incorporar o caráter substitutivo do modelo de assistência ${ }^{21}$. E que mesmo nos locais onde se alcançou a universalidade do acesso, este está geralmente associado às atividades programáticas, com prejuízo do acolhimento das demandas espontâneas ${ }^{18,27}$.

Dificuldades para superar o modelo assistencial tradicional e avançar em direção a um modelo mais inclusivo de assistência foi objetivo de pesquisa qualitativa envolvendo 190 profissionais de PS/AMA e UBS que mostrou que a concepção limitada sobre o papel das UBS, sobretudo de profissionais que atuam na atenção primária, resulta, possivelmente, em práticas restritivas ao acesso da população ${ }^{28}$. 0 estudo citado revela um aspecto importante do acesso, remetendoo à falta de conhecimento dos profissionais sobre a concepção do modelo de atenção preconizado no SUS. Outro estudo, realizado no interior paulista, analisou os fatores associados ao acesso aos serviços básicos de saúde e aponta que estes se associam às experiências anteriores de atendimento no serviço e no imaginário construído pela clientela sobre o atendimento ${ }^{29}$.

Seja por experiência negativa anterior dos pais ou por desconhecimento a respeito do funcionamento do sistema, os usuários do SUS continuam buscando os serviços de emergência para tentar resolver problemas de saúde de baixa complexidade clínica e o fazem no período diurno, preferencialmente vespertino, horário em que as unidades de saúde da atenção primária estão em funcionamento.

O horário de maior pico de atendimento no PSI, encontrado neste estudo sugere algumas situações, tais como: a tentativa frustrada de atendimento na atenção primária, o cuidado em alimentar a criança antes de buscar atendimento, já conhecendo o tempo de espera no pronto socorro ou ainda, a possibilidade de ter recebido uma comunicação da escola infantil ou creche, solicitando providências dos pais para buscar a criança que manifesta algum sinal ou sintoma de doença.

\section{CONCLUSÃO}

0 estudo mostrou que a capacidade de acolhimento de crianças e adolescentes dos serviços de atenção básica foi majoritariamente moderada (55,5\%), com melhor desempenho das unidades que atuam na Estratégia Saúde da Família.

Recomenda-se a realização de novas pesquisas que aprofundem os aspectos associados à baixa capacidade de acolhimento e os principais fatores contributivos da não reorientação no modelo assistencial, conforme recomendam as políticas públicas de saúde. 


\section{REFERÊNCIAS}

1. Almeida PF, Giovanella L. Avaliação em Atenção Básica à Saúde no Brasil: mapeamento e análise das pesquisas realizadas e/ou financiadas pelo Ministério da Saúde entre os anos de 2000 e 2006. Cad Saúde Pública. 2008; 24(8):1727-42.

2. Tesser CD, Norman AH. Repensando o acesso ao cuidado na Estratégia Saúde da Família. Saúde Soc. 2014; 23(3):869-83.

3. Nascimento APS, Santos LF, Carnut L. Atenção primária à saúde via estratégia de saúde da família no Sistema Único de Saúde: introdução aos problemas inerentes à operacionalização de suas ações. J Manag Prim Health Care. 2011; 2(1):18-24.

4. Lentsck MH, Kluthcovsky ACGC, Kluthcovsky FA. Avaliação do Programa Saúde da Família: uma revisão. Ciênc Saúde Coletiva. 2010; 15(Supl. 3):3455-66.

5. Vanderlei MIG, Almeida MCP. A concepção e prática dos gestores e gerentes da estratégia de saúde da família. Ciênc Saúde Coletiva. 2007; 12(2):443-53.

6. Giovanella L, Mendonça MHM. Atenção primária à saúde. In: Giovanella L, Escorel S, Lobato LVC, Noronha JC, Carvalho AI., organizadores. Políticas e sistemas de saúde. Rio de Janeiro: Fiocruz; 2008.

7. Ministério da Saúde (Br). Política Nacional de Atenção Básica. Brasília (DF): Secretaria de Atenção Básica; Departamento de Atenção à Saúde; 2008.

8. Ministério da Saúde (Br). Acolhimento à demanda espontânea na atenção básica. Brasília (DF): Ministério da Saúde; 2011. (Cadernos de Atenção Básica, n.28, v.1).

9. Ministério da Saúde (Br). Implementação da rede de atenção às urgências/emergências. Brasília (DF): Ministério da Saúde; 2011.

10. Ministério da Saúde (Br). Acolhimento e classificação de risco nos serviços de urgência. Brasília (DF): Ministério da Saúde; 2009.

11. Souza CC, Chianca LM, Diniz AS, Chianca CM. Principais queixas de pacientes de urgência segundo o protocolo de classificação de risco de Manchester. Rev Enferm UFPE. 2012; 6(3):540-8.
12. Souza ECF, Vilar RLA, Rocha NPD, Uchoa AC, Rocha PM. Acesso e acolhimento na atenção básica: uma análise da percepção dos usuários e profissionais de saúde. Cad Saúde Pública. 2008; 24(1):100-10.

13. Rocha AS, Vilar RLA, Melo RHV, França RCS. 0 acolhimento na atenção básica em saúde: relações de reciprocidade entre trabalhadores e usuários. Saúde Debate. 2015; 39(104):11423.

14. Ministério da Saúde (Br). Acolhimento à demanda espontânea: queixas mais comuns na atenção básica. Brasília (DF): Ministério da Saúde; 2013. (Cadernos de Atenção Básica, $n$. 28; v.2).

15. Traldi MC. As Políticas públicas de saúde e os principais desafios para a administração de enfermagem em saúde coletiva. In: Santos AS, Traldi MC, organizadores. Administração de enfermagem em saúde coletiva. Barueri: Manole; 2015.

16. Farias DC, Celino SDM, Peixoto JBS, Mayara Lima Barbosa ML, Costa GMC. Acolhimento e resolubilidade das urgências na estratégia saúde da família. Rev Bras Educ Med. 2015; 39(1):79-87.

17. Silva JA. Reorganizar e humanizar o processo de trabalho através do acolhimento: relato de experiência. Rev Baiana Saúde Pública. 2015; 39(1):174-81.

18. Silva LA, Casotti CA, Chaves SCL. A produção científica brasileira sobre a Estratégia Saúde da Família e a mudança no modelo de atenção. Ciênc Saúde Coletiva. 2013; 18(1):221-32.

19. Camargo Junior KR, Campos EAS, Bustamante-Teixeira MT, Mascarenhas MTM, Mauad NM, FrancoTB, et al. Avaliação da atenção básica pela ótica político-institucional e da organização da atenção com ênfase na integralidade. Cad Saúde Pública. 2008; 24(Supl.1):58-68.

20. Finkler AL,Viera CS, Tacla MT, Toso BR. 0 acesso e a dificuldade na resolutividade do cuidado da criança na atenção primária à saúde. Acta Paul Enferm. 2014; 27(6):548-53. 21. Coutinho LRP, Barbieri AR, Santos MLM. Acolhimento na atenção primária à saúde: 
revisão integrativa. Saúde Debate. 2015; 39(105):514-24.

22. Gomes GP, Moulaz ALS, Pereira DL, Sá GB, Chaveiro ND, Santos TR. A análise do acolhimento na perspectiva das equipes de saúde da família e dos usuários no Centro de Saúde da Família 04 do Riacho Fundo II. Rev APS. 2014; 17(3):325-33.

23. Lopes AS, Vilar RLA, Melo RHV, França RCS. 0 acolhimento na atenção básica em saúde: relações de reciprocidade entre trabalhadores e usuários. Saúde Debate. 2015; 39(104):11423.

24. Mitre SM, Andrade EIG, Cotta RMM. Avanços e desafios do acolhimento na operacionalização e qualificação do Sistema Único de Saúde na atenção primária: um resgate da produção bibliográfica do Brasil. Ciênc Saúde Coletiva. 2012; 17(8):2071-85.

25. Viegas APB, Carmo RF, Luz ZMP. Fatores que influenciam o acesso aos serviços de saúde na visão de profissionais e usuários de uma unidade básica de referência. Saúde Soc. 2015; 24(1):100-12.

26. Simons DA. Avaliação do perfil da demanda na unidade de emergência em Alagoas a partir da municipalização da saúde e do Programa
Saúde da Família. [Doutorado]. Recife: Centro de Pesquisas Aggeu Magalhães, Fundação Oswaldo Cruz; 2008.

27. Tesser CD, Norman AH. Repensando o acesso ao cuidado na Estratégia de Saúde da Família. Saúde Soc. 2014; 23(3):869-83.

28. Puccini PT, Cornetta VK, Sahyom TZ, Fuentes ICP, Botta LMG, Puccini RF. Concepção de profissionais de saúde sobre o papel das unidades básicas nas redes de atenção do SUS/Brasil. Ciênc Saúde Coletiva. 2012; 17(11):2941-52.

29. Bonello AALM, Corrêa CRS. Acesso aos serviços de saúde e fatores associados: estudo de base populacional. Ciênc Saúde Coletiva. 2014; 19(11):4397-406.

CONTRIBUIÇÕES
Maria Cristina Traldi foi responsável pela
concepção do projeto de pesquisa, análise e
discussão dos resultados e na elaboração do
artigo. Laís Rabesco participou na concepção
do projeto de pesquisa, na coleta e analise dos
dados. Márcia Regina Campos Costa da
Fonseca atuou na análise dos dados e na
revisão do artigo.

\section{Como citar este artigo (Vancouver):}

Traldi MC, Rabesco L, Fonseca MRCC. Avaliação da capacidade de acolhimento da demanda espontânea nos serviços de atenção básica. REFACS [Online]. 2016 [citado em (inserir dia, mês e ano de acesso)]; 4(2). Disponível em: (link de acesso). DOI: 10.18554/refacs.v4i2.1641.

Como citar este artigo (ABNT):

TRALDI, M. C.; RABESCO, L.; FONSECA, M. R. C. C. Avaliação da capacidade de acolhimento da demanda espontânea nos serviços de atenção básica. REFACS, Uberaba, MG, v. 4, n. 2, p. 107-118, 2016. Disponível em: (link de acesso). DOI: 10.18554/refacs.v4i2.1641. Acesso em: (inserir dia, mês e ano de acesso).

Como citar este artigo (APA):

Trald,i M. C., Rabesco, L. \& Fonseca, M. R. C. C. (2016). Avaliação da capacidade de acolhimento da demanda espontânea nos serviços de atenção básica. REFACS, 4(2), 107-118. Recuperado em (dia), (mês), (ano) de (link de acesso). DOI: 10.18554/refacs.v4i2.1641. 